\title{
New CORAVEL spectroscopic-binary orbits of giant barium stars. II ${ }^{\star}$
}

\author{
S. Udry ${ }^{1}$, M. Mayor ${ }^{1}$, S. Van Eck ${ }^{1,2}$, A. Jorissen ${ }^{2}$, L. Prévot $^{3}$, S. Grenier ${ }^{4}$, and H. Lindgren ${ }^{5}$ \\ 1 Observatoire de Genève, CH-1290 Sauverny, Switzerland \\ 2 Institut d'Astronomie et d'Astrophysique, Université Libre de Bruxelles, C.P. 226, Bd. du Triomphe, B-1050 Bruxelles, \\ Belgium \\ 3 Observatoire de Marseille, 2 Place Le Verrier, F-13248 Marseille, France \\ 4 Observatoire de Paris-Meudon, D.A.S.G.A.L., URA 335, F-92195 Meudon Cedex, France \\ ${ }^{5}$ Lund Observatory, Box 43, S-22100 Lund, Sweden
}

Received December 15, 1997; accepted January 29, 1998

\begin{abstract}
This paper complements the set of spectroscopic orbits for giant barium stars given in Udry et al. (1998) and provides data for 20 binaries (18 orbits +2 minimum-period determinations).
\end{abstract}

Key words: stars: late-type - stars: chemically peculiar — binaries: spectroscopic

\section{Introduction}

In this paper, we present the second part of a set of spectroscopic orbits of giant barium stars, complementary to the orbits described in a companion paper (Udry et al. 1998, Paper I). The overall sample comes from a systematic survey undertaken in the framework of a collaboration between the Geneva and Bruxelles groups to gain insight into the formation process of barium and Tc-poor S stars. The binary nature of these stars accounts for the observed chemical peculiarities through mass transfer across the system. This sample also allows us to confirm the evolutionary link between barium and Tc-poor S stars. These points are addressed in a companion paper providing the complete analysis of the overall sample (Jorissen et al. 1998).

Other CORAVEL users, around LP in Marseille, also interested in some of the same stars, joined in to permit a better determination of the orbital parameters and thus take the best advantage of the available measurements. The join binary sample is presented here.

Send offprint requests to: S. Udry

* Based on observations obtained at the Haute-Provence Observatory (France) and at the European Southern Observatory (ESO, La Silla, Chile).
As the star sample and the observation characteristics are described in details in Paper I, we will just briefly summarize the main related information in Sect. 2. Section 3 provides the derived radial-velocity curves and orbital elements. Some individual stars are commented as well.

\section{Samples and observations}

\subsection{Barium-star samples}

The two samples of barium stars with strong and mild anomalies (Ba4 or $\mathrm{Ba} 5$ and $\mathrm{Ba}<1$, $\mathrm{Ba} 1$ or $\mathrm{Ba} 2$, respectively, on the scale defined by Warner 1965) to which belong the binaries presented in this paper were taken from the list of Lü et al. (1983). The strong barium stars were followed to address the question of whether binarity is a necessary condition to form a strong barium star. The mild barium stars were selected to provide a comparison set. The samples are described in more details in Tables 1 and 2 of Paper I.

For the strong barium sample, 29 stars were followed yielding 29 spectroscopic orbits. Eight of them are described in Sect. 3, 18 in Paper I, 2 in Griffin et al. (1996) and 1 in Jorissen et al. (1995). For the mild barium set, 12 binaries are presented in Sect. 3 whereas 14 may be found in Paper I and 1 in Griffin (1991).

The individual measurements will be available at the Centre de Données Stellaires (CDS) in Strasbourg or on our dedicated web page:

obswww.unige.ch/ udry/cine/barium/barium.html.

\subsection{Observations}

Starting back in 1984, the observations were performed with the two CORAVEL spectrometers (Baranne et al. 
Table 1. Orbital elements for the strong barium stars. $N$ is the number of measurements used to derive the orbital solution and $\mathrm{O}-\mathrm{C}$ the residue around this solution. $\Delta T$ is the span of the observations

\begin{tabular}{|c|c|c|c|c|c|c|c|c|c|c|c|}
\hline $\begin{array}{l}\text { Id } \\
\text { HD } \\
\end{array}$ & $\begin{array}{c}P \\
\text { [days] }\end{array}$ & $\begin{array}{c}T \text { [HJD } \\
-2400000] \\
\end{array}$ & $e$ & {$\left[\begin{array}{c}\gamma \\
{\left[\mathrm{kms}^{-1}\right]}\end{array}\right.$} & $\begin{array}{c}\omega \\
{[\mathrm{deg}]}\end{array}$ & $\begin{array}{c}K \\
{\left[\mathrm{~km} \mathrm{~s}^{-1}\right]}\end{array}$ & $f(m)$ & $\begin{array}{l}a \sin i \\
{[\mathrm{Gm}]}\end{array}$ & $N$ & $\begin{array}{c}\mathrm{O}-\mathrm{C} \\
\left.\mathrm{km} \mathrm{s}^{-1}\right]\end{array}$ & $\begin{array}{c}\Delta T \\
{[\text { days }]}\end{array}$ \\
\hline 43389 & $\begin{array}{r}1689.03 \\
8.760\end{array}$ & $\begin{array}{r}47222.46 \\
61.25\end{array}$ & $\begin{array}{l}0.082 \\
0.016\end{array}$ & $\begin{array}{r}53.14 \\
0.08\end{array}$ & $\begin{array}{r}189.50 \\
12.77\end{array}$ & $\begin{array}{l}6.26 \\
0.12\end{array}$ & $\begin{array}{l}4.267 \mathrm{e}-02 \\
2.476 \mathrm{e}-03\end{array}$ & $\begin{array}{r}144.990 \\
2.892\end{array}$ & 24 & 0.351 & 3350 \\
\hline 44896 & $\begin{array}{r}628.889 \\
0.886\end{array}$ & $\begin{array}{r}48464.30 \\
28.41\end{array}$ & $\begin{array}{l}0.025 \\
0.010\end{array}$ & $\begin{array}{r}52.16 \\
0.06\end{array}$ & $\begin{array}{r}227.04 \\
16.27\end{array}$ & $\begin{array}{l}9.04 \\
0.07\end{array}$ & $\begin{array}{l}.827 \mathrm{e}-02 \\
1.091 \mathrm{e}-03\end{array}$ & $\begin{array}{r}78.187 \\
0.598\end{array}$ & 19 & 0.209 & 3270 \\
\hline 46407 & $\begin{array}{r}457.403 \\
0.140\end{array}$ & $\begin{array}{r}47677.45 \\
40.73\end{array}$ & $\begin{array}{l}0.013 \\
0.008\end{array}$ & $\begin{array}{r}-3.45 \\
0.05\end{array}$ & $\begin{array}{l}73.73 \\
32.08\end{array}$ & $\begin{array}{l}9.03 \\
0.07\end{array}$ & $\begin{array}{l}3.503 \mathrm{e}-02 \\
8.655 \mathrm{e}-04\end{array}$ & $\begin{array}{r}56.825 \\
0.468\end{array}$ & 68 & 0.396 & 5883 \\
\hline 50082 & $\begin{array}{r}2896.034 \\
21.339\end{array}$ & $\begin{array}{r}45953.12 \\
56.58\end{array}$ & $\begin{array}{l}0.188 \\
0.022\end{array}$ & $\begin{array}{r}-17.37 \\
0.07\end{array}$ & $\begin{array}{r}205.61 \\
6.61\end{array}$ & $\begin{array}{l}4.54 \\
0.11\end{array}$ & $\begin{array}{l}2.676 \mathrm{e}-02 \\
1.998 \mathrm{e}-03\end{array}$ & $\begin{array}{r}177.777 \\
4.593\end{array}$ & 29 & 0.345 & 5543 \\
\hline 92626 & $\begin{array}{r}918.188 \\
1.193\end{array}$ & $\begin{array}{r}49147.83 \\
2.06\end{array}$ & $\begin{array}{l}0.000 \\
0.011\end{array}$ & $\begin{array}{r}16.28 \\
0.06\end{array}$ & $\begin{array}{r}0.00 \\
36.85\end{array}$ & $\begin{array}{l}7.64 \\
0.09\end{array}$ & $\begin{array}{l}4.249 \mathrm{e}-02 \\
1.531 \mathrm{e}-03\end{array}$ & $\begin{array}{r}96.436 \\
1.164\end{array}$ & 35 & 0.315 & 3803 \\
\hline 107541 & $\begin{array}{r}3569.924 \\
46.120\end{array}$ & $\begin{array}{r}44388.16 \\
124.56\end{array}$ & $\begin{array}{l}0.104 \\
0.031\end{array}$ & $\begin{array}{r}88.15 \\
0.08\end{array}$ & $\begin{array}{r}223.09 \\
12.23\end{array}$ & $\begin{array}{l}4.32 \\
0.12\end{array}$ & $\begin{array}{l}2.940 \mathrm{e}-02 \\
2.555 \mathrm{e}-03\end{array}$ & $\begin{array}{r}210.901 \\
6.629\end{array}$ & 16 & 0.284 & $\begin{array}{l}3973 \\
\end{array}$ \\
\hline 201657 & $\begin{array}{r}1710.414 \\
15.023\end{array}$ & $\begin{array}{r}46154.95 \\
103.55\end{array}$ & $\begin{array}{l}0.171 \\
0.072\end{array}$ & $\begin{array}{r}-27.66 \\
0.18\end{array}$ & $\begin{array}{r}272.52 \\
22.51\end{array}$ & $\begin{array}{l}2.86 \\
0.22\end{array}$ & $\begin{array}{l}3.966 \mathrm{e}-03 \\
9.471 \mathrm{e}-04\end{array}$ & $\begin{array}{r}66.228 \\
5.300\end{array}$ & 15 & 0.287 & 4481 \\
\hline 211594 & $\begin{array}{r}1018.861 \\
2.709\end{array}$ & $\begin{array}{r}48538.19 \\
38.80\end{array}$ & $\begin{array}{l}0.058 \\
0.015\end{array}$ & $\begin{array}{r}-9.88 \\
0.05\end{array}$ & $\begin{array}{l}73.65 \\
13.89\end{array}$ & $\begin{array}{l}5.10 \\
0.06\end{array}$ & $\begin{array}{l}1.399 \mathrm{e}-02 \\
5.376 \mathrm{e}-04\end{array}$ & $\begin{array}{r}71.381 \\
0.931\end{array}$ & 49 & 0.333 & 5030 \\
\hline
\end{tabular}

1979), on the 1-m Swiss telescope at the Haute-Provence Observatory for the northern sky and on the 1.54m Danish telescope at La Silla (Chile, ESO) for the southern sky. The distributions of individual errors $\left(\overline{\varepsilon_{i}}=0.3 \mathrm{~km} \mathrm{~s}^{-1}\right)$, number of measurements $(\bar{N}=19)$ and time span $(\overline{\Delta T}=3398 \mathrm{~d})$ per star are displayed in Fig. 1 of Paper I. They attest the large observational effort dedicated to these programmes and the good quality and homogeneity of the measurements.

More detailed information on the scientific background of the different projects, on the sample selection strategies, or on the observational characteristics of the measurements can be found in Jorissen et al. (1998) and in Paper I as also in Gòmez et al. (1997) and Menessier et al. (1997).

\section{Radial-velocity curves and orbital parameters}

The same presentation strategy as in Paper I is adopted. The orbital elements and the phase-folded radial-velocity curves are provided for the binaries with stable orbital solutions. Badly constrained parameters are readily identifiable by their large uncertainties. In case the period has to be fixed to obtain a satisfactory orbit, it is fixed to a minimized value determined by the minimum residue around the obtained solution. In such a case, no uncertainty is given for the fixed period. When the star is clearly binary but the period is not sufficiently covered to derive a preliminary orbit, only a minimum period is given. The radial velocities in function of Julian dates are then displayed.

Figure 1 and Table 1 provide the results for the strong barium sample ( 8 orbits) whereas Fig. 2 and Table 2 give the information for the mild barium binaries (10 orbits + 2 minimum-period binaries).
For the uncompletely-covered star HD 53199, the period has been fixed to a minimized value. In a few cases (see Jorissen et al. 1998), even though the Lucy-Sweeney test was compatible with a circular orbital solution (Lucy \& Sweeney 1971), the free-eccentricity solution has been given because their is no physical argument to prefer circular orbits in the case of barium stars. Two stars (HD 46407 and HD 223617) have an orbital solution derived from a combined set of CORAVEL measurements and radial velocities obtained at the Dominion Astrophysical Observatory (DAO) by McClure \& Woodsworth (1990). The fair number of new CORAVEL measurements allows us to slightly improve the previously published orbits.

Finally, the star HD 101079 deserves a comment. Only 7 CORAVEL radial-velocity measurements are available to date. They permit to derive two equally-probable orbital solutions with periods of $1588 \mathrm{~d}$ and $3120 \mathrm{~d}$. In the coming months, new measurements will allow us to decide between the two solutions but for the moment only a minimum period $P>1550 \mathrm{~d}$ is given in Table 2 .

Acknowledgements. This research has been supported partly by the Fonds National de la Recherche Scientifique (Switzerland, Belgium) and the University of Geneva (Geneva Observatory).

\section{References}

Baranne A., Mayor M., Poncet J.L., 1979, Vistas Astron. 23, 279

Gòmez A.E., Luri X., Grenier S., et al., 1997, A\&A 319, 881

Griffin R.F., 1991, The Observatory 111, 29

Griffin R.F., Jorissen A., Mayor M., 1996, The Observatory 116,298

Jorissen A., Hennen O., Mayor M., Bruch A., Sterken C., 1995, A\&A 301, 707 

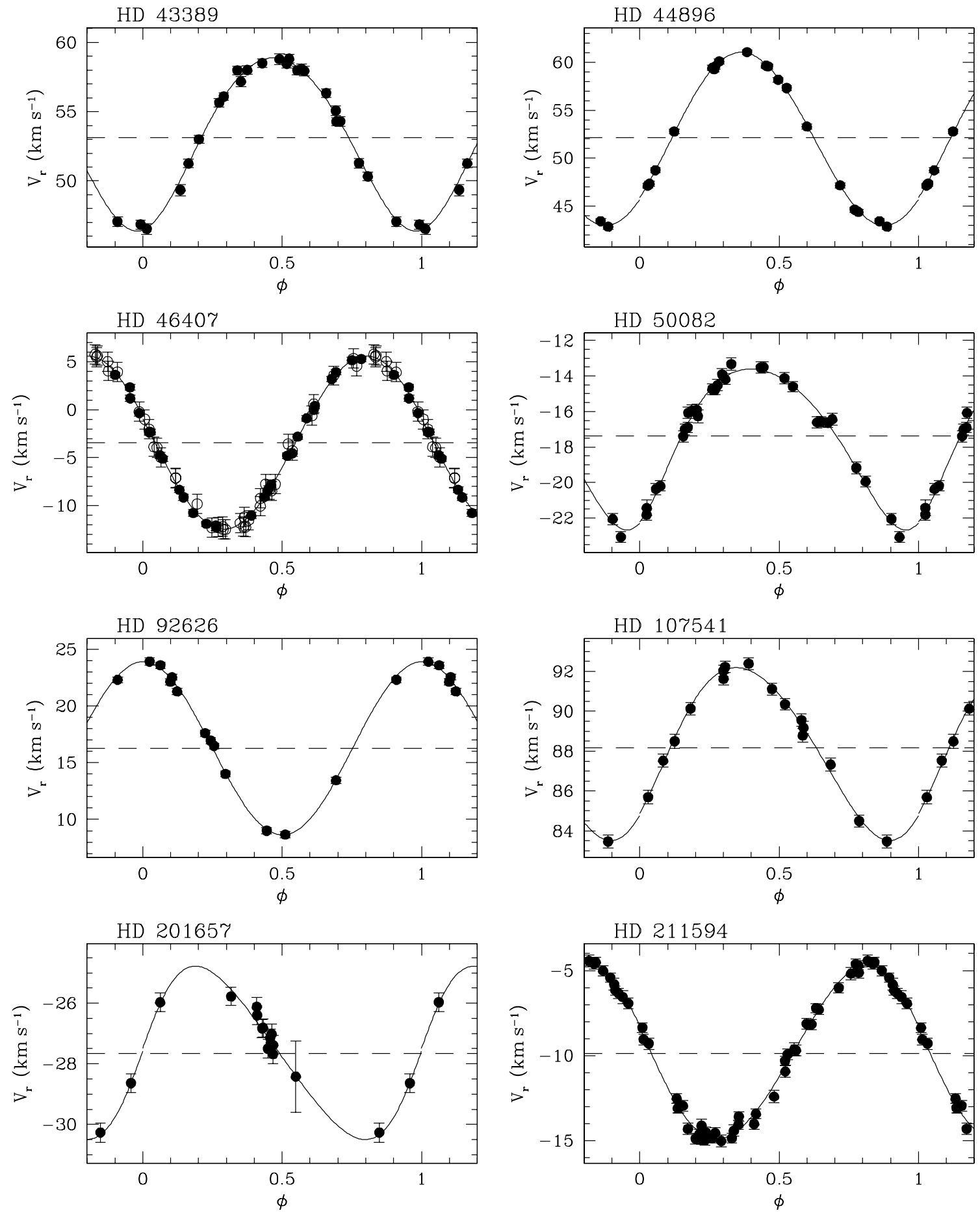

Fig. 1. Phase-folded radial-velocity curves for the strong barium stars. Open circles are for DAO measurements

Jorissen A., Van Eck S., Mayor M., Udry S., 1998, A\&A (in press)

Lü P.K., Dawson D.W., Upgren A.R., Weis E.W., 1983, ApJS 52, 169

Lucy L.B., Sweeney M.A., 1971, AJ 76, 544
McClure R.D., Woodsworth A.W., 1990, ApJ 352, 709

Mennessier M.O., Luri X., Figueras S., 1997, A\&A 326, 722

Udry S., Mayor M., Van Eck S., Jorissen A., 1998, A\&AS 131, 25 (Paper I)

Warner B., 1965, MNRAS 129, 263 

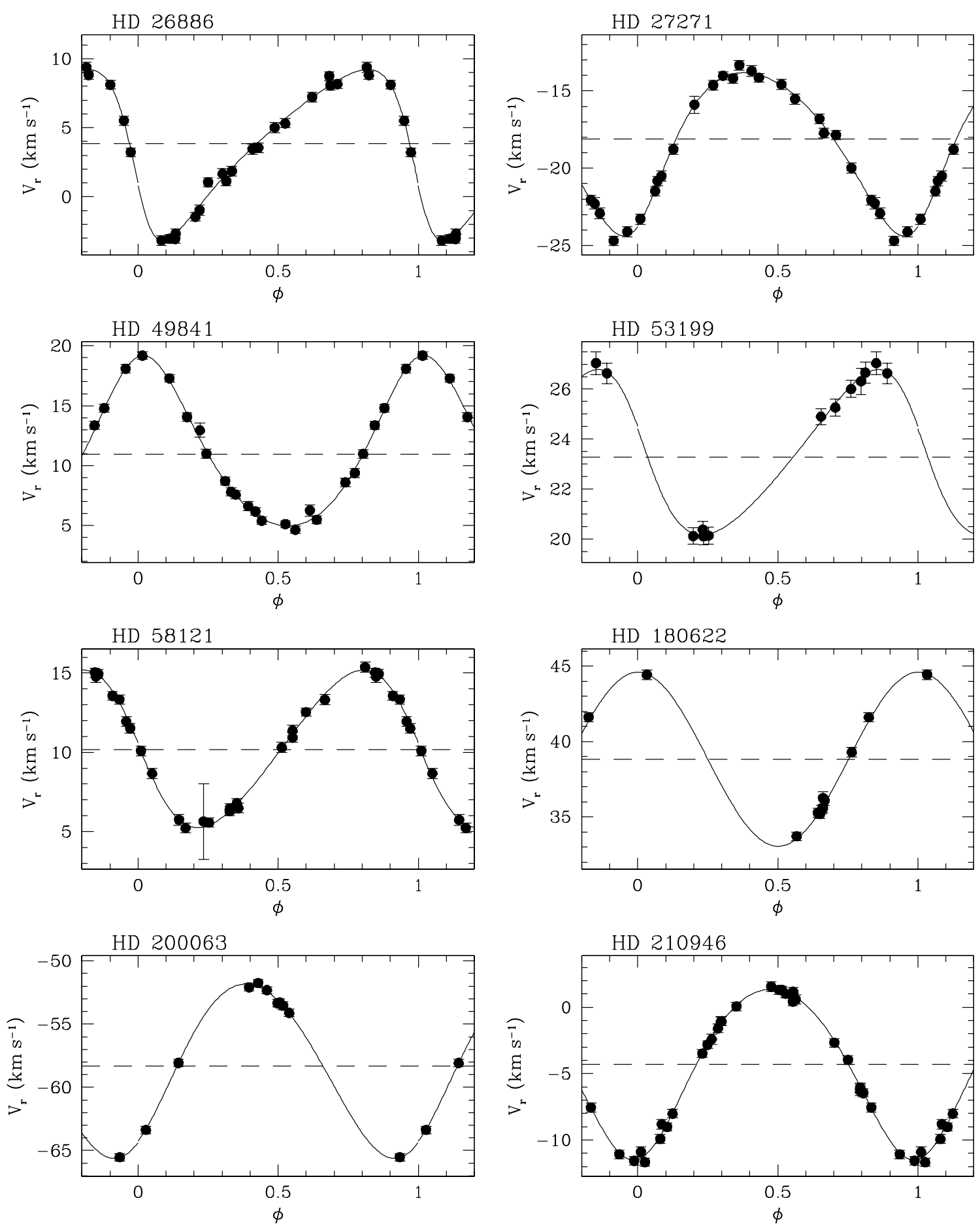

Fig. 2. Phase-folded radial-velocity curves for the mild barium stars. Open circles are for DAO measurements. Due to the noncomplete coverage of the orbit a minimized period has been fixed for HD 53199. Long-period stars without orbital solution have their radial velocities displayed as a function of Julian dates 

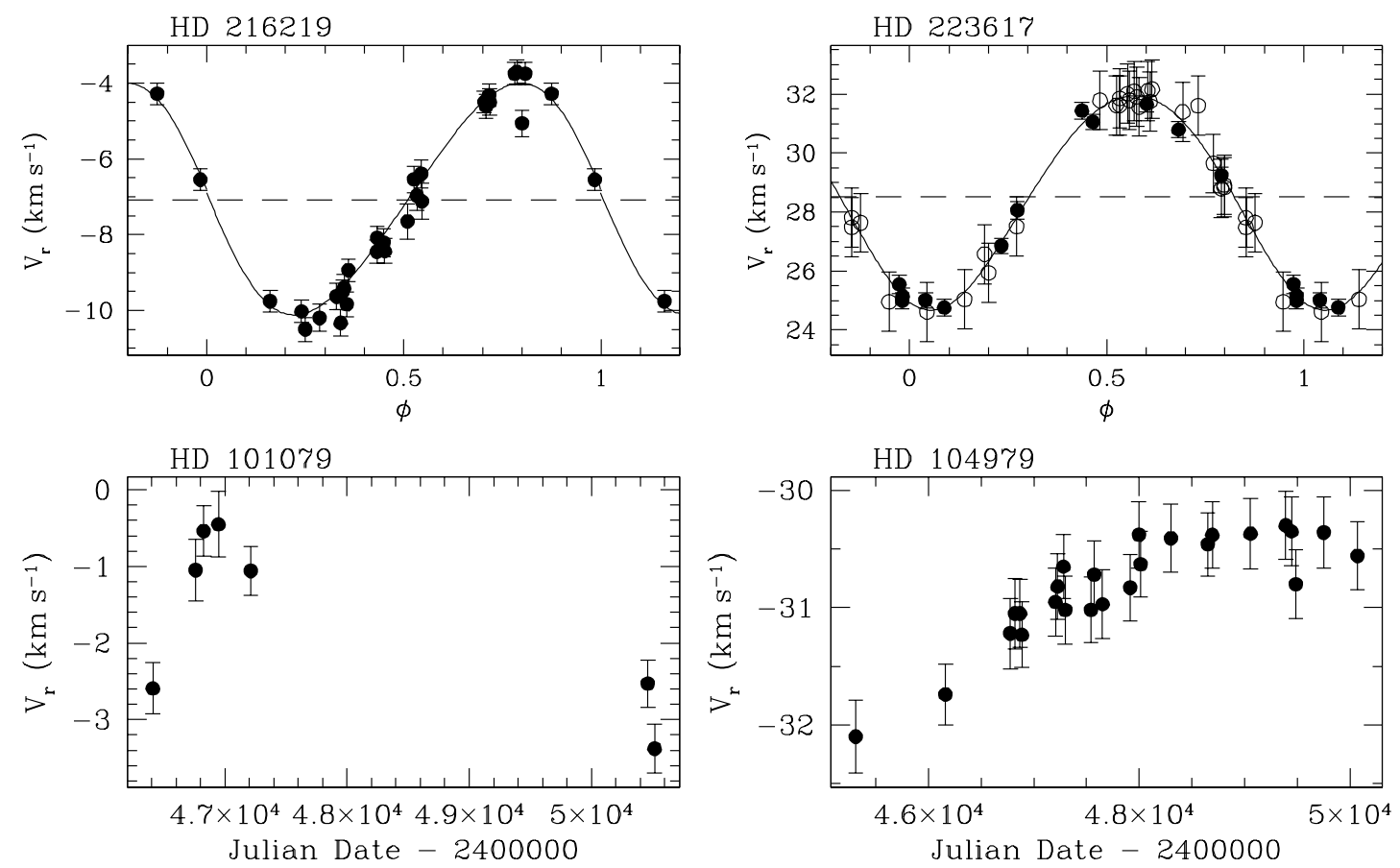

Fig. 2. continued

Table 2. Orbital elements for the mild barium stars. No uncertainties are given for fixed parameters

\begin{tabular}{|c|c|c|c|c|c|c|c|c|c|c|c|}
\hline $\begin{array}{c}\text { Id } \\
\text { HD }\end{array}$ & $\begin{array}{c}P \\
\text { [days] }\end{array}$ & $\begin{array}{c}T \text { [HJD } \\
-2400000] \\
\end{array}$ & $e$ & {$\left[\begin{array}{c}\gamma \\
{\left[\mathrm{kms}^{-1}\right]}\end{array}\right.$} & $\begin{array}{c}\omega \\
{[\mathrm{deg}]}\end{array}$ & $\begin{array}{c}K \\
{\left[\mathrm{~km} \mathrm{~s}^{-1}\right]}\end{array}$ & $f(m)$ & $\begin{array}{l}a \sin i \\
{[\mathrm{Gm}]}\end{array}$ & $\mathrm{N}$ & $\begin{array}{c}\mathrm{O}-\mathrm{C} \\
\left.\mathrm{km} \mathrm{s}^{-1}\right]\end{array}$ & $\begin{array}{c}\Delta T \\
{[\text { days] }}\end{array}$ \\
\hline 26886 & $\begin{array}{r}1263.230 \\
3.729\end{array}$ & $\begin{array}{r}48952.12 \\
9.23\end{array}$ & $\begin{array}{l}0.395 \\
0.025\end{array}$ & $\begin{array}{l}3.85 \\
0.09\end{array}$ & $\begin{array}{r}110.83 \\
3.35\end{array}$ & $\begin{array}{l}6.24 \\
0.17\end{array}$ & $\begin{array}{l}2.471 \mathrm{e}-02 \\
2.161 \mathrm{e}-03\end{array}$ & $\begin{array}{r}99.580 \\
2.915\end{array}$ & 23 & 0.400 & 4356 \\
\hline 27271 & $\begin{array}{r}1693.838 \\
9.061 \\
\end{array}$ & $\begin{array}{r}7104.38 \\
19.45 \\
\end{array}$ & $\begin{array}{l}0.217 \\
0.021 \\
\end{array}$ & $\begin{array}{r}-18.10 \\
0.07 \\
\end{array}$ & $\begin{array}{r}208.83 \\
4.68 \\
\end{array}$ & $\begin{array}{l}5.28 \\
0.10 \\
\end{array}$ & $\begin{array}{l}2.406 \mathrm{e}-02 \\
1.425 \mathrm{e}-03 \\
\end{array}$ & $\begin{array}{r}120.016 \\
2.445 \\
\end{array}$ & 23 & 0.310 & 3350 \\
\hline 49841 & $\begin{array}{r}897.098 \\
1.823 \\
\end{array}$ & $\begin{array}{r}8339.71 \\
13.51 \\
\end{array}$ & $\begin{array}{l}0.161 \\
0.015 \\
\end{array}$ & $\begin{array}{r}10.95 \\
0.08 \\
\end{array}$ & $\begin{array}{r}350.73 \\
5.47 \\
\end{array}$ & $\begin{array}{l}7.12 \\
0.12 \\
\end{array}$ & $\begin{array}{l}3.239 \mathrm{e}-02 \\
1.693 \mathrm{e}-03 \\
\end{array}$ & $\begin{array}{r}86.739 \\
1.520 \\
\end{array}$ & 21 & 0.331 & 3431 \\
\hline 53199 & $\begin{array}{c}7500.0 \\
-\end{array}$ & $\begin{array}{r}41116.20 \\
62.94\end{array}$ & $\begin{array}{l}0.212 \\
0.225\end{array}$ & $\begin{array}{r}23.27 \\
0.06\end{array}$ & $\begin{array}{r}73.12 \\
1.59\end{array}$ & $\begin{array}{l}3.31 \\
0.07\end{array}$ & $\begin{array}{l}2.645 \mathrm{e}-02 \\
1.570 \mathrm{e}-03\end{array}$ & $\begin{array}{r}333.996 \\
6.608\end{array}$ & 11 & 0.174 & 4492 \\
\hline 58121 & $\begin{array}{r}1214.257 \\
5.712\end{array}$ & $\begin{array}{r}46811.21 \\
23.25\end{array}$ & $\begin{array}{l}0.140 \\
0.015\end{array}$ & $\begin{array}{r}10.18 \\
0.05\end{array}$ & $\begin{array}{r}86.83 \\
7.62\end{array}$ & $\begin{array}{l}4.97 \\
0.07\end{array}$ & $\begin{array}{l}1.500 \mathrm{e}-02 \\
6.856 \mathrm{e}-04\end{array}$ & $\begin{array}{r}82.125 \\
1.303\end{array}$ & 23 & 0.236 & 3033 \\
\hline 101079 & $>1550$ & & & & & & & & 7 & & 4107 \\
\hline 104979 & $>4700$ & & & & & & & & 25 & & 4764 \\
\hline 180622 & $\begin{array}{r}4049.166 \\
37.709\end{array}$ & 50534.41 & $\begin{array}{l}0.061 \\
0.097\end{array}$ & $\begin{array}{r}39.18 \\
1.17\end{array}$ & $\begin{array}{l}251.45 \\
184.26\end{array}$ & $\begin{array}{l}5.52 \\
0.54\end{array}$ & $\begin{array}{l}7.026 \mathrm{e}-02 \\
2.053 \mathrm{e}-02\end{array}$ & $\begin{array}{r}306.681 \\
29.991\end{array}$ & 10 & 0.255 & 4482 \\
\hline 200063 & $\begin{array}{r}1735.451 \\
8.166\end{array}$ & $\begin{array}{r}47744.64 \\
109.53\end{array}$ & $\begin{array}{l}0.073 \\
0.037\end{array}$ & $\begin{array}{r}-58.32 \\
0.25\end{array}$ & $\begin{array}{r}216.25 \\
21.77\end{array}$ & $\begin{array}{l}6.89 \\
0.17\end{array}$ & $\begin{array}{l}5.849 \mathrm{e}-02 \\
4.299 \mathrm{e}-03\end{array}$ & $\begin{array}{r}169.999 \\
4.083\end{array}$ & 10 & 0.234 & 4483 \\
\hline 210946 & $\begin{array}{r}1529.544 \\
4.058\end{array}$ & $\begin{array}{r}46578.18 \\
24.23\end{array}$ & $\begin{array}{l}0.126 \\
0.011\end{array}$ & $\begin{array}{r}-4.28 \\
0.05\end{array}$ & $\begin{array}{r}186.98 \\
5.86\end{array}$ & $\begin{array}{l}6.43 \\
0.07\end{array}$ & $\begin{array}{l}.132 \mathrm{e}-02 \\
1.448 \mathrm{e}-03\end{array}$ & $\begin{array}{r}134.265 \\
1.604\end{array}$ & 30 & 0.264 & 3642 \\
\hline 216219 & $\begin{array}{r}4098.036 \\
111.540\end{array}$ & $\begin{array}{r}44824.92 \\
339.24\end{array}$ & $\begin{array}{l}0.101 \\
0.043\end{array}$ & $\begin{array}{r}-7.17 \\
0.12\end{array}$ & $\begin{array}{l}82.32 \\
23.84\end{array}$ & $\begin{array}{l}3.11 \\
0.11\end{array}$ & $\begin{array}{l}1.261 \mathrm{e}-02 \\
1.395 \mathrm{e}-03\end{array}$ & $\begin{array}{r}174.383 \\
7.835\end{array}$ & 29 & 0.368 & 4486 \\
\hline 223617 & $\begin{array}{r}1293.699 \\
3.894\end{array}$ & $\begin{array}{r}47276.68 \\
89.83\end{array}$ & $\begin{array}{l}0.061 \\
0.020\end{array}$ & $\begin{array}{r}28.51 \\
0.06\end{array}$ & $\begin{array}{r}157.61 \\
25.27\end{array}$ & $\begin{array}{l}3.63 \\
0.08\end{array}$ & $\begin{array}{l}6.381 \mathrm{e}-03 \\
4.075 \mathrm{e}-04\end{array}$ & $\begin{array}{r}64.426 \\
1.383\end{array}$ & 39 & 0.344 & 5843 \\
\hline
\end{tabular}

